\title{
Article
}

\section{Increased Incidence and Associated Risk Factors of Aspergillosis in Patients with Bronchiectasis}

\author{
Bumhee Yang ${ }^{1,+}$, Taehee Kim ${ }^{2,+}$, Jiin Ryu ${ }^{3,+}{ }^{+}$, Hye Yun Park ${ }^{4}$, Bin Hwangbo ${ }^{5}$, Sun-Young Kong ${ }^{6}(\mathbb{D}$, \\ Yong-Soo Kwon ${ }^{7}$, Seung Jun Lee ${ }^{8} \sqrt{1}$, Seung Won Ra ${ }^{9}$, Yeon-Mok Oh ${ }^{10}$, Jang Won Sohn ${ }^{11}$, Kang Hyeon Choe ${ }^{1}$, \\ Hayoung Choi $2, * \mathbb{C}$ and Hyun Lee $11, * \mathbb{C}$
}

1 Division of Pulmonary and Critical Care Medicine, Department of Internal Medicine, Chungbuk National University Hospital, College of Medicine, Chungbuk National University, Cheongju 28644, Korea; ybhworld0415@gmail.com (B.Y.); choekh@chungbuk.ac.kr (K.H.C.)

2 Division of Pulmonary, Allergy, and Critical Care Medicine, Department of Internal Medicine, Hallym University Kangnam Sacred Heart Hospital, Hallym University College of Medicine, Seoul 07441, Korea; sosom02@naver.com

3 Biostatistical Consulting and Research Lab, Medical Research Collaborating Center, Hanyang University, Seoul 04763, Korea; ryu3209@hanyang.ac.kr

4 Division of Pulmonary and Critical Care Medicine, Department of Medicine, Samsung Medical Center, School of Medicine, Sungkyunkwan University, Seoul 06351, Korea; hyeyunpark@skku.edu

5 Division of Pulmonology, Center of Lung Cancer, Hospital, National Cancer Center, Goyang 10408, Korea; hbb@ncc.re.kr

6 Department of Laboratory Medicine, Hospital, National Cancer Center, Goyang 10408, Korea; ksy@ncc.re.kr

7 Department of Internal Medicine, Chonnam National University Hospital, Gwangju 61469, Korea; yskwon@chonnam.ac.kr

check for updates

Citation: Yang, B.; Kim, T.; Ryu, J.; Park, H.Y.; Hwangbo, B.; Kong, S.-Y.; Kwon, Y.-S.; Lee, S.J.; Ra, S.W.; Oh, Y.-M.; et al. Increased Incidence and Associated Risk Factors of Aspergillosis in Patients with Bronchiectasis. J. Pers. Med. 2021, 11, 422. https://doi.org/10.3390/ jpm11050422

Academic Editor: Klaus Bønnelykke

Received: 28 March 2021

Accepted: 13 May 2021

Published: 17 May 2021

Publisher's Note: MDPI stays neutral with regard to jurisdictional claims in published maps and institutional affiliations.

Copyright: (c) 2021 by the authors. Licensee MDPI, Basel, Switzerland. This article is an open access article distributed under the terms and conditions of the Creative Commons Attribution (CC BY) license (https:/ / creativecommons.org/licenses/by/ $4.0 /)$.
8 Division of Pulmonology and Allergy, Department of Internal Medicine, Gyeongsang National University Hospital, School of Medicine, Gyeongsang National University, Jinju 52727, Korea; juny2278@naver.com

9 Division of Pulmonary Medicine, Department of Internal Medicine, Ulsan University Hospital, College of Medicine, University of Ulsan, Ulsan 44033, Korea; skydocra@gmail.com

10 Department of Pulmonary and Critical Care Medicine, Asan Medical Center, College of Medicine, University of Ulsan, Seoul 05505, Korea; yeonmok.oh@gmail.com

11 Division of Pulmonary Medicine and Allergy, Department of Internal Medicine, Hanyang University College of Medicine, Seoul 04763, Korea; jwsohn@hanyang.ac.kr

* Correspondence: hychoimd@gmail.com (H.C.); namuhanayeyo@hanyang.ac.kr (H.L.)

$+\quad$ These authors contributed equally to this work.

Abstract: There are insufficient data regarding the relationship between non-cystic fibrosis bronchiectasis and incident aspergillosis. We performed a population-based, matched cohort study using data from the Korean National Health Insurance database between 2003 and 2013. The incidence of aspergillosis was 50/100,000 person-years in the bronchiectasis cohort and 11/100,000 person-years in the matched cohort (subdistribution hazard ratio, 4.53; 95\% confidence interval (CI), 3.25-6.32). Among the bronchiectasis cohort, chronic obstructive pulmonary disease (adjusted HR, 1.95; 95\% CI, 1.07-3.57), previous pulmonary tuberculosis (adjusted HR, 3.67; 95\% CI, 2.03-6.64), and non-tuberculous mycobacterial pulmonary disease (adjusted HR, 11.25; 95\% CI, 1.49-85.18) increased the risk of incident aspergillosis. The incidence of aspergillosis in patients with bronchiectasis was approximately 4.5 -fold that in those without bronchiectasis. Comorbid pulmonary diseases-chronic obstructive pulmonary disease, previous pulmonary tuberculosis, and non-tuberculous mycobacterial pulmonary disease-significantly increased the risk of aspergillosis in patients with bronchiectasis. Our study indicates that close monitoring is warranted for aspergillosis in patients with bronchiectasis.

Keywords: aspergillosis; bronchiectasis; epidemiology; risk factors

\section{Introduction}

Non-cystic fibrosis bronchiectasis (hereafter referred to as bronchiectasis) is a chronic lung disease characterised by permanent bronchial dilatation, variable mucociliary clear- 
ance, and recurrent infections [1,2]. The inherent dysfunctional mucociliary clearance leads to persistent bacterial infection, chronic inflammation of the bronchial tree, and progressive tissue destruction [3]. Therefore, patients with bronchiectasis are susceptible to many infections, such as bacterial and non-tuberculous mycobacterium (NTM) infections [4-6]. In addition to these bacteria, filamentous Aspergillus species, the most common fungi isolated from the sputum of patients with bronchiectasis, are also recognised as potential pathogens in bronchiectasis [3].

Of a number of manifestations of Aspergillus disease encountered in patients with bronchiectasis, the most common comorbid infectious conditions include pulmonary aspergilloma, chronic pulmonary aspergillosis (CPA), and invasive aspergillosis [7-9]. Considering that patients with bronchiectasis with these types of aspergillosis may benefit from treatments such as antifungal treatment, it is important to elucidate whether bronchiectasis increases the risk of aspergillosis and to identify risk factors related to the process. However, there is no concrete evidence of this issue.

The identification of risk factors predicting comorbid conditions in patients with bronchiectasis is important for establishing strategies to monitor and prevent these conditions. It would also facilitate early therapeutic intervention when aspergillosis develops in patients with bronchiectasis. However, little information is available regarding the risk factors for aspergillosis in patients with bronchiectasis, except for allergic bronchopulmonary aspergillosis (ABPA). Thus, elucidation of these risk factors is urgently needed.

In this study, we aimed to investigate whether patients with bronchiectasis are at a higher risk of aspergillosis than those without bronchiectasis and to identify risk factors for incident aspergillosis in patients with bronchiectasis.

\section{Materials and Methods}

\subsection{Data Source}

We used the National Health Insurance Service-National Sample Cohort (NHIS-NSC), a population-based retrospective cohort that included a $2.2 \%$ representative sample of all Korean citizens. The NHIS-NSC database includes health data regarding (1) major and minor diagnoses in the form of the 10th revision of the International Statistical Classification of Diseases and Related Health Problems (ICD-10) codes, and (2) procedures and drug prescriptions in outpatient clinics, emergency departments, and hospitalisations, in addition to age, sex, and type of insurance [10].

\subsection{Study Population}

As shown in Figure 1, between 1 January 2003 and 31 December 2012, the database included data for 861,808 adult patients aged $\geq 20$ years. After exclusion of patients with human immunodeficiency virus infection $(n=627)$ and cystic fibrosis $(n=88)$, the sample included 17,679 patients with bronchiectasis and 843,414 without bronchiectasis. Among the 17,679 patients with bronchiectasis, we excluded those diagnosed with aspergillosis within 1 year after enrolment $(n=90)$ and those who died within 1 year after enrolment $(n=683)$. Among 843,414 patients without bronchiectasis, we excluded those diagnosed with aspergillosis within 1 year after enrolment in the cohort $(n=56)$ and those who died within 1 year after enrolment in the cohort $(n=4663)$. To establish a matched cohort, we performed a 1:4 matching for age and sex for each bronchiectasis patient and controls without bronchiectasis. The aspergillosis-naive bronchiectasis cohort $(n=16,906)$ and the aspergillosis-naive matched cohort $(n=67,624)$ were followed until 31 December 2013 to compare the incidence of aspergillosis between them. As the NHIS-NSC database contains all claim data of participants during the study period (2002-2013), only deceased or emigrated participants were excluded from the dataset [10]. None of the patients had a follow-up duration of less than 1 year. 


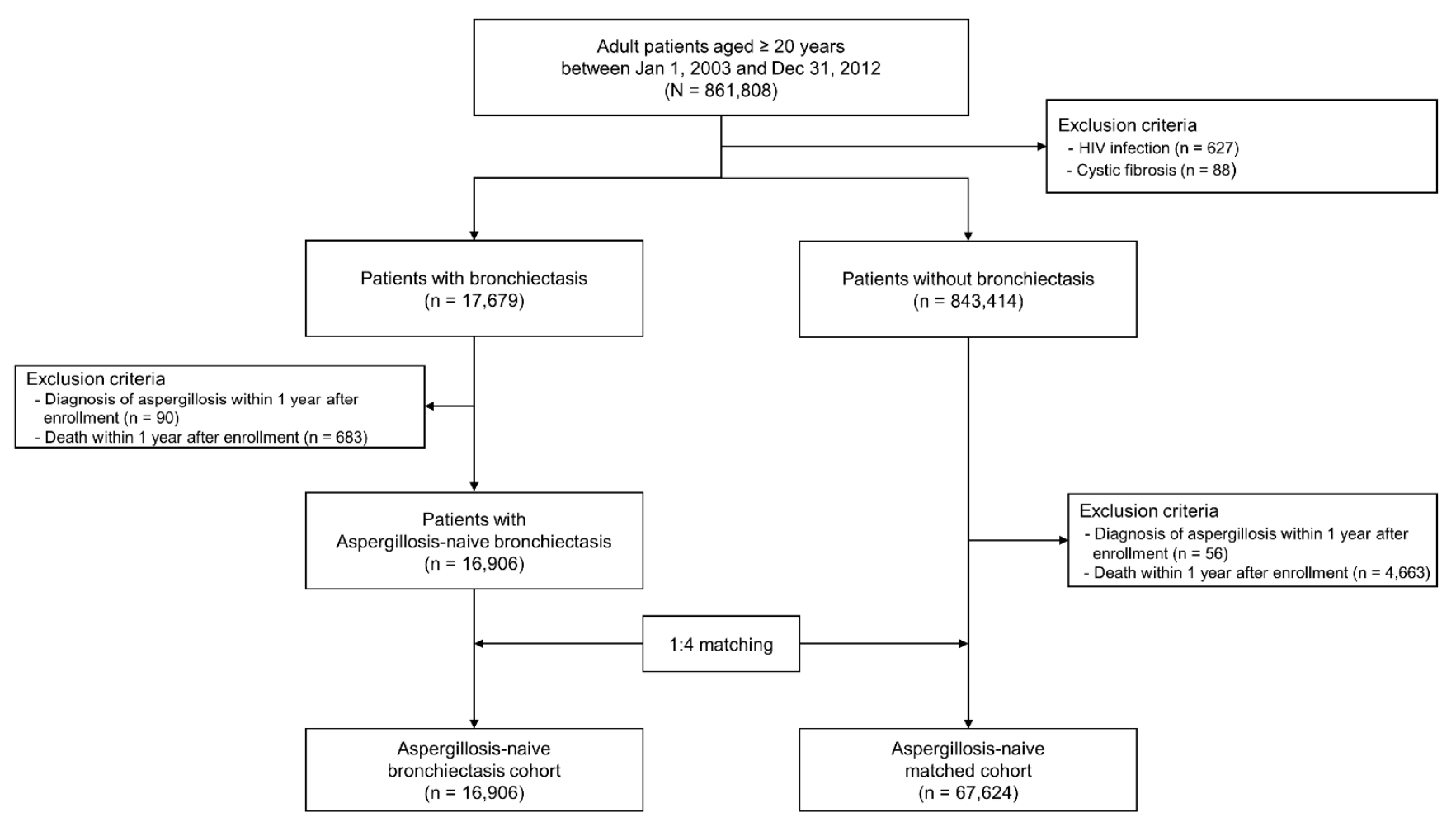

Figure 1. Flow chart of the study. HIV, human immunodeficiency virus.

The study protocol was approved by the Institutional Review Board of Chungbuk National University Hospital (IRB application no. 2020-12-027). The requirement for informed consent from the participants was waived because the NHIS database was constructed after anonymization.

\subsection{Definitions}

As exposure of this study, adult bronchiectasis was defined by the following criteria: (1) age $\geq 20$ years and (2) at least one claim under ICD-10 diagnosis code J47. Patients with cystic fibrosis (E84) and human immunodeficiency virus infection (B20-B24) were excluded from this study.

As an outcome of this study, aspergillosis was defined by the presence of a primary or secondary diagnostic code associated with aspergillosis (B44). Patients with tonsillar aspergillosis (B44.2) or ABPA (B44.81) were excluded.

Bronchiectasis-associated comorbidities were also defined using the following ICD-10 diagnosis codes: chronic obstructive pulmonary disease (COPD) (J42-J44, except J43.0 (unilateral emphysema)), asthma (J45-J46), pulmonary tuberculosis (TB) (A15-A19), nontuberculous mycobacterial pulmonary disease (NTM-PD) (A31 and claims data for acidfast bacilli smear or mycobacterial culture), diabetes mellitus (E10-E14), rheumatologic disease (M05-M06, M315, M32-M34, M351, M353, and M360), and malignancy (C00-C97), including lung cancer (C34) [11,12].

Regarding corticosteroid use, the study population was categorised into three groups according to the use of inhaled corticosteroids (ICS) (no ICS use, ICS use for less than 1 year, and ICS use for at least 1 year) and systemic corticosteroids (no systemic corticosteroid use, $<10 \mathrm{mg}$ /day prednisolone equivalent dose, and $>10 \mathrm{mg}$ /day prednisolone equivalent dose) [13].

\subsection{Statistical Analysis}

The McNemar test was used to compare baseline characteristics between the bronchiectasis cohort and the matched cohort. To construct the aspergillosis-naive bronchiectasis cohort, we excluded patients diagnosed with aspergillosis within 1 year after enrolment 
in the bronchiectasis cohort. Given that 1 year might not be enough to construct an aspergillosis-naive bronchiectasis cohort, we performed a sensitivity analysis with different exclusion criteria using 2- and 3-year washout periods.

We compared the incidence rate (per 100,000 person-years (PY)) of aspergillosis between the two cohorts using the normal approximation test for binominals. The cumulative incidence of aspergillosis was compared between the bronchiectasis cohort and the matched cohort using the cumulative incidence curve and Gray's test. To evaluate the impact of bronchiectasis on incident aspergillosis in the bronchiectasis cohort relative to the matched cohort, a proportional subdistribution hazards model for aspergillosis with death as a competing event was used.

To analyse the risk factors associated with aspergillosis, we performed Cox proportional hazards regression models. In multivariable models, variables with $p<0.05$ in univariable analysis were included: age, asthma, COPD, previous pulmonary TB, NTM-PD, use of ICS, and systemic corticosteroid use. All tests were two-tailed, and $p$-values $<0.05$ were considered statistically significant. All statistical analyses were performed using SAS ${ }^{\circledR}$ software (version 9.4; SAS Institute, Cary, NC, USA).

\section{Results}

\subsection{Baseline Characteristics}

As shown in Table 1, there were no significant intergroup differences between the bronchiectasis and matched cohorts in age and sex. The proportion of patients who received medical aid was higher in the bronchiectasis cohort than in the matched cohort $(2.9 \%$ vs. $2.3 \%, p<0.001)$. Regarding pulmonary comorbidities, the proportions of asthma $(34.4 \%$ vs. $8.9 \%)$, COPD (27.7\% vs. $5.2 \%)$, previous pulmonary TB (12.0\% vs. $2.6 \%)$, and NTM-PD $(0.1 \%$ vs. $<0.1 \%)$ were higher in the bronchiectasis cohort than in the matched cohort ( $p<0.001$ for all). Regarding extrapulmonary comorbidities, diabetes mellitus (20.4\% vs. $15.8 \%)$, rheumatologic diseases (6.1\% vs. $3.4 \%)$, lung cancer ( $3.8 \%$ vs. $0.3 \%)$, and other cancers $(3.7 \%$ vs. $2.2 \%)$ were significantly more frequent in the bronchiectasis cohort than in the matched cohort $(p<0.001$ for all).

\subsection{Incidence Rate and Risk of Aspergillosis in the Bronchiectasis Cohort Versus the Matched Cohort}

During the median follow-up duration of 6.1 (interquartile range, 3.4-8.7) years, the age- and sex-adjusted incidence of aspergillosis was 50.2/100,000 PY in the bronchiectasis cohort and 10.9/100,000 PY in the matched cohort (subdistribution hazard ratio (sdHR), 4.53; 95\% confidence interval (CI), 3.25-6.32). The risk of aspergillosis was higher in patients with bronchiectasis aged $<60$ years (sdHR, 4.91; 95\% CI, 3.07-7.84) than in those aged $\geq 60$ years (sdHR, 4.17; 95\% CI, 2.60-6.71) ( $p$ for interaction $<0.01$ ). The risk for incident aspergillosis was higher among females in the bronchiectasis cohort (sdHR, 5.33; 95\% CI, 3.21-8.87) than among males in the bronchiectasis cohort (sdHR, 4.00; 95\% CI, 2.57-6.23) ( $p$ for interaction < 0.01) (Table 2). Sensitivity analyses also revealed comparable HRs for incident aspergillosis in patients with bronchiectasis relative to those without bronchiectasis: 2-year (sdHR, 4.94; 95\% CI, 3.16-7.71) and 3-year (sdHR, 4.94; 95\% CI, 3.02-8.08) washout periods, respectively (Supplementary Table S1).

As shown in Figure 2, the cumulative incidence of aspergillosis was significantly higher in the bronchiectasis cohort than in the matched cohort (Gray's test, $p<0.001$ ). Subgroup analyses also revealed that the cumulative incidence of aspergillosis was significantly higher in the bronchiectasis cohort than in the matched cohort, regardless of age group or sex (Gray's test, $p<0.001$ for all subgroups, Supplementary Figure S1). Detailed information on the diagnosis of aspergillosis in the bronchiectasis cohort and the matched cohort are summarised in Supplementary Table S2. 
Table 1. Baseline characteristics of patients.

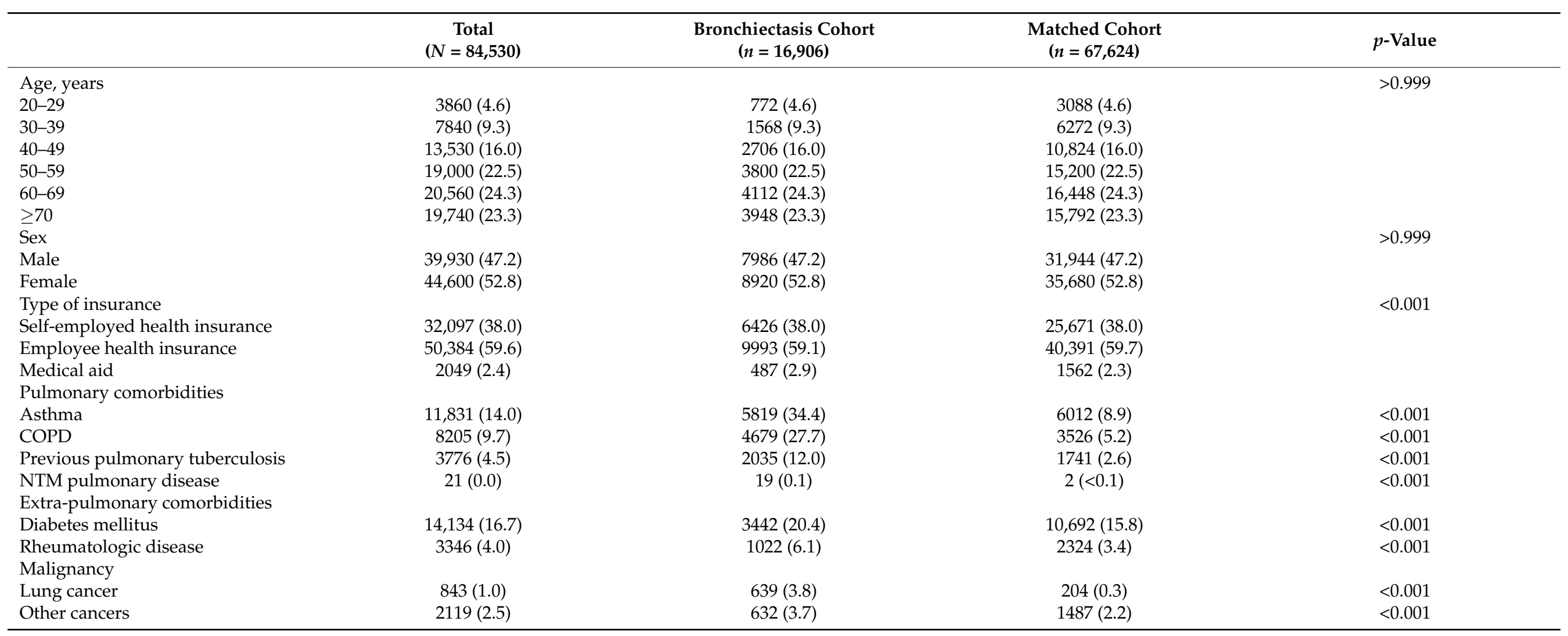

Data are presented as number (percentage). Abbreviations: COPD, chronic obstructive lung disease; NTM, non-tuberculous mycobacteria. 
Table 2. Sex- and age-specific incidence rates (/100,000 person-years) and subdistribution hazard ratios of aspergillosis in the bronchiectasis cohort relative to the matched cohort.

\begin{tabular}{|c|c|c|c|c|c|c|c|c|c|c|c|c|}
\hline & \multicolumn{4}{|c|}{ Total $(N=84,675)$} & \multicolumn{4}{|c|}{ Male $(n=40,015)$} & \multicolumn{4}{|c|}{ Female $(n=44,660)$} \\
\hline & Case & IR & sdHR & $95 \% \mathrm{CI}$ & Case & IR & sdHR & $95 \%$ CI & Case & IR & sdHR & $95 \%$ CI \\
\hline \multicolumn{13}{|l|}{ Overall } \\
\hline Matched & 45 & 10.9 & Ref & & 27 & 14.2 & Ref & & 18 & 8.1 & Ref & \\
\hline Bronchiectasis & 51 & 50.2 & 4.53 & $3.25-6.32$ & 27 & 57.9 & 4.00 & $2.57-6.23$ & 24 & 43.7 & 5.33 & $3.21-8.87$ \\
\hline \multicolumn{13}{|l|}{$\begin{array}{l}\text { Age group } \\
<60 \text { years }\end{array}$} \\
\hline Matched & 22 & 9.5 & Ref & & 16 & 15.0 & Ref & & 6 & 4.8 & Ref & \\
\hline $\begin{array}{l}\text { Bronchiectasis } \\
\geq 60 \text { years }\end{array}$ & 27 & 46.8 & 4.91 & $3.07-7.84$ & 13 & 49.1 & 3.25 & $1.77-5.97$ & 14 & 44.9 & 9.33 & $4.11-21.22$ \\
\hline Matched & 23 & 12.8 & Ref & & 11 & 13.1 & Ref & & 12 & 12.5 & Ref & \\
\hline Bronchiectasis & 24 & 54.7 & 4.17 & $2.60-6.71$ & 14 & 69.3 & 5.09 & $2.64-9.82$ & 10 & 42.2 & 3.33 & $1.66-6.69$ \\
\hline
\end{tabular}

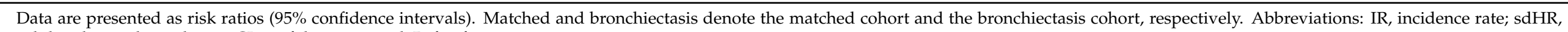
subdistribution hazard ratio; CI, confidence interval; Ref, reference. 


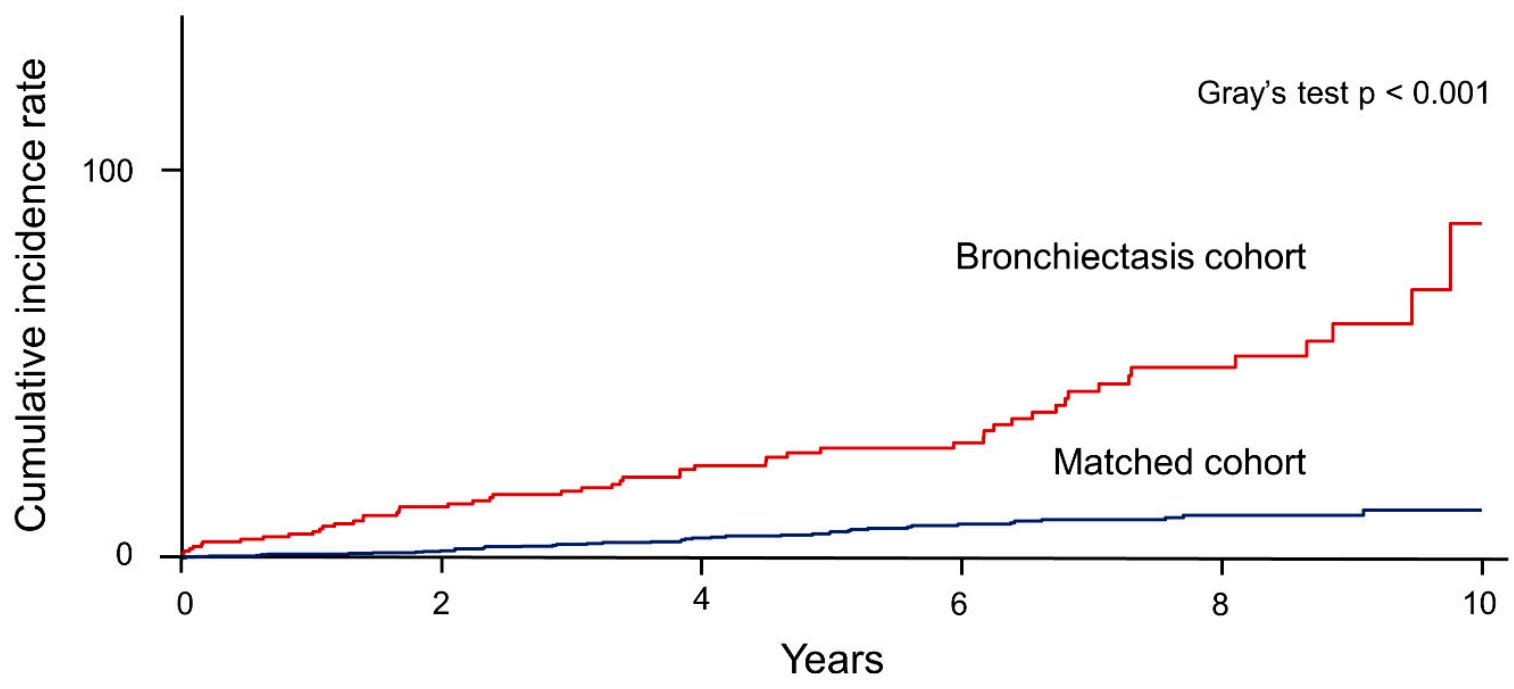

Figure 2. Cumulative incidence of aspergillosis (/100,000 person-years) in the bronchiectasis and matched cohorts.

\subsection{Risk Factors of Aspergillosis in the Bronchiectasis Cohort}

Table 3 summarises the risk factors for aspergillosis in the bronchiectasis cohort. Although age was significantly associated with an increased risk of aspergillosis in the bronchiectasis cohort in univariable analysis, this association was not statistically significant in the multivariable analysis. Multivariable analysis revealed that pulmonary comorbidities-COPD (adjusted hazard ratio (HR), 1.95; 95\% CI, 1.07-3.57), previous pulmonary TB (adjusted HR, 3.67; 95\% CI, 2.03-6.64), and NTM-PD (adjusted HR, 11.25; $95 \%$ CI, 1.49-85.18)—significantly increased the risk of aspergillosis. 
Table 3. Risk factors for aspergillosis in patients with bronchiectasis.

\begin{tabular}{|c|c|c|c|c|c|c|}
\hline & \multirow{2}{*}{$\begin{array}{l}\text { Numbers at Risk } \\
\quad(N=16,906)\end{array}$} & \multirow{2}{*}{$\begin{array}{c}\text { Aspergillosis } \\
\quad(n=51)\end{array}$} & \multicolumn{2}{|c|}{ Univariable Analysis } & \multicolumn{2}{|c|}{ Multivariable Analysis } \\
\hline & & & HR & $95 \% \mathrm{CI}$ & Adjusted HR & $95 \% \mathrm{CI}$ \\
\hline \multicolumn{7}{|l|}{ Age } \\
\hline$\leq 39$ & $2340(13.8)$ & $2 / 2340(0.1)$ & Ref & Ref & Ref & Ref \\
\hline $40-49$ & $2706(16.0)$ & $11 / 2706(0.4)$ & 4.91 & $1.09-22.16$ & 4.43 & $0.98-20.01$ \\
\hline $50-59$ & $3800(22.5)$ & $14 / 3800(0.4)$ & 5.30 & $1.20-23.34$ & 4.01 & $0.90-17.81$ \\
\hline $60-69$ & $4112(24.3)$ & $13 / 4112(0.3)$ & 4.57 & $1.03-20.27$ & 2.77 & $0.61-12.52$ \\
\hline$\geq 70$ & $3948(23.4)$ & $11 / 3948(0.3)$ & 5.32 & $1.17-24.06$ & 3.16 & $0.68-14.66$ \\
\hline \multicolumn{7}{|l|}{$\overline{\text { Sex }}$} \\
\hline Female & $7986(47.2)$ & $27 / 7986(0.3)$ & Ref & Ref & & \\
\hline Male & $8920(52.8)$ & $24 / 8920(0.3)$ & 1.35 & $0.78-2.34$ & & \\
\hline \multicolumn{7}{|l|}{ Type of insurance } \\
\hline $\begin{array}{l}\text { Self-employed health } \\
\text { insurance }\end{array}$ & $6426(38.0)$ & $19 / 6426(0.3)$ & Ref & Ref & & \\
\hline Employee health insurance & $9993(59.1)$ & $31 / 9993(0.3)$ & 1.16 & $0.66-2.06$ & & \\
\hline Medical aid & $487(2.9)$ & $1 / 487(0.2)$ & 1.73 & $0.23-13.02$ & & \\
\hline \multicolumn{7}{|l|}{ Comorbidities } \\
\hline COPD & $4679(27.7)$ & $27 / 4679(0.6)$ & 3.09 & $1.78-5.35$ & 1.95 & $1.07-3.57$ \\
\hline Asthma & $5819(34.4)$ & $28 / 5819(0.5)$ & 2.27 & $1.31-3.93$ & 1.27 & $0.68-2.37$ \\
\hline NTM pulmonary disease & $19(0.1)$ & $1 / 19(5.3)$ & 28.73 & $3.96-208.41$ & 11.25 & $1.49-85.18$ \\
\hline Diabetes mellitus & $3442(20.4)$ & $11 / 3442(0.3)$ & 1.32 & $0.68-2.58$ & & \\
\hline Rheumatologic disease & $1022(6.1)$ & $3 / 1022(0.3)$ & 0.93 & $0.29-2.99$ & & \\
\hline Lung cancer & $639(3.8)$ & $3 / 639(0.5)$ & 1.65 & $0.52-5.31$ & & \\
\hline \multicolumn{7}{|l|}{ Medication } \\
\hline \multicolumn{7}{|l|}{ Use of ICS } \\
\hline No use & $13,149(77.8)$ & $27 / 13,149(0.2)$ & Ref & Ref & Ref & Ref \\
\hline$<1$ year & $2540(15.0)$ & $14 / 2540(0.6)$ & 2.50 & $1.31-4.77$ & 1.83 & $0.92-3.63$ \\
\hline$\geq 1$ year & $1217(7.2)$ & $10 / 1217(0.8)$ & 3.39 & $1.64-7.01$ & 1.69 & $0.74-3.89$ \\
\hline \multicolumn{7}{|l|}{ Systemic corticosteroids * } \\
\hline No use & $3602(21.3)$ & $5 / 3602(0.1)$ & Ref & Ref & Ref & Ref \\
\hline$<10 \mathrm{mg} /$ day & $12,764(75.5)$ & $41 / 12,764(0.3)$ & 1.43 & $0.56-3.65$ & 1.29 & $0.50-3.31$ \\
\hline$\geq 10 \mathrm{mg} /$ day or more & $540(3.2)$ & $5 / 540(0.9)$ & 3.60 & $1.04-12.50$ & 2.15 & $0.59-7.86$ \\
\hline
\end{tabular}

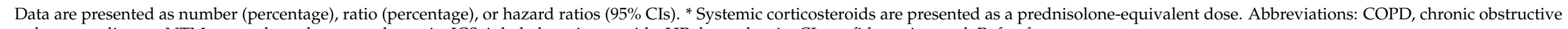
pulmonary disease; NTM, non-tuberculous mycobacteria; ICS, inhaled corticosteroids; HR, hazard ratio; CI, confidence interval; Ref, reference. 


\section{Discussion}

In the present study, we compared the incidence of aspergillosis between patients with bronchiectasis and age- and sex-matched patients without bronchiectasis. Our results show that the risk of aspergillosis in patients with bronchiectasis was approximately 4.5 -fold higher than that in those without bronchiectasis. Compared to patients without bronchiectasis, the risk of aspergillosis in those with bronchiectasis was especially higher in those aged $<60$ years and women. Regarding risk factors, COPD, previous pulmonary TB, and NTM-PD significantly increased the risk of aspergillosis in patients with bronchiectasis.

To the best of our knowledge, this is the first study to assess the incidence and risk factors of incident aspergillosis in patients with bronchiectasis using a nationally representative cohort. Aspergillus infections, including Aspergillus bronchitis and CPA, are not rare in patients with bronchiectasis, and it has been suggested that they either cause bronchiectasis or complicate existing bronchiectasis $[9,14]$. However, the burden of aspergillosis among patients with bronchiectasis has not been well studied as the focus of previous studies was mainly limited to ABPA and did not include comorbid aspergillosis [11,15-17]. Data regarding the relationship between bronchiectasis and Aspergillus infection were considered in studies that evaluated bronchiectasis as a comorbid pulmonary disease in patients with aspergillosis. In these studies, the proportion of patients with bronchiectasis was $38 \%$ among patients with CPA and $14.5 \%$ among patients with pulmonary aspergillosis $[18,19]$. However, because of their cross-sectional design, these previous studies could not clarify whether there is a cause-and-effect link between these two conditions. The present study has a substantial advantage in terms of causal inference in that approximately $0.3 \%$ of bronchiectasis cases without aspergillosis develop aspergillosis during a follow-up duration of about six years.

In this study, the risk of aspergillosis in patients with bronchiectasis compared to those without bronchiectasis was particularly higher among younger patients (less than 60 years) and females. In younger patients, the prevalence of comorbid conditions (e.g., diabetes mellitus and malignancy), which increases susceptibility to aspergillosis, was lower than in the elderly population. Thus, the effect of bronchiectasis on the development of aspergillosis might be more influential in younger patients than in older patients. In line with our findings, a previous study showed that the positive rates of A. fumigatus-specific immunoglobulin $\mathrm{G} \mathrm{Ab}$ were higher in young and female patients among patients with TB [12]. The present study and a previous study suggest that the risk of aspergillosis is higher in women than in men when structural bronchial abnormalities, such as bronchiectasis and TB sequelae, are present. Accordingly, our results may have important clinical implications, indicating that more attention is needed regarding surveillance for aspergillosis development among younger or female patients with bronchiectasis. However, except for the current study, no studies have evaluated the susceptibility to aspergillosis by age or sex in patients with bronchiectasis compared to those without bronchiectasis. Thus, further studies are needed to confirm our findings.

We evaluated the risk factors of aspergillosis in patients with bronchiectasis, and our results provided valuable information for clinicians assessing the risk of aspergillosis in patients with bronchiectasis with respect to comorbid conditions, including COPD, TB, and NTM-PD. COPD is a chronic inflammatory disease of the airways [20], and airway colonisation by Aspergillus species is a common feature of COPD [21]. Thus, COPD patients have been recognised to be at an increased risk of pulmonary aspergillosis [22]. Additionally, some previous studies reported that COPD is an underlying pulmonary comorbid condition in $42-67 \%$ of patients with pulmonary aspergillosis [23,24]. In agreement with previous studies, we found that COPD increased the risk of aspergillosis 2 -fold in patients with bronchiectasis.

A significant association between CPA development and post-TB patients was shown in a cohort study in the 1960s; among 544 TB patients with persistent cavities after treatment, $25 \%$ had positive Aspergillus precipitins in blood samples, and $14 \%$ had aspergillomas in radiological images [25]. CPA is one of the most common infectious sequelae in post-TB 
patients, with an estimated prevalence of 1.2 million cases annually worldwide, indicating a significant, newly emerging global disease burden [26]. Sehgal et al. also showed a significant occurrence of Aspergillus sensitisation and chronic infection in patients with bronchiectasis, especially in those with post-TB bronchiectasis [27]. However, the associations of post-TB sequelae, bronchiectasis, and pulmonary aspergillosis are complicated as these conditions interact. Conflicting results have also been reported in a previous study. A study performed in Uganda found that approximately $5 \%$ of post-TB patients were diagnosed with CPA during a 2-year follow-up period. Although bronchiectasis was present in $86 \%$ of patients, it was not associated with an increased risk of CPA [28]. In our study, we observed that previous TB history increased the risk of aspergillosis 4-fold in patients with bronchiectasis. After TB treatment, residual lung damage in the form of bronchiectasis may lead to chronic Aspergillus colonisation and sensitisation, followed by aspergillosis infection. Hence, clinicians should be aware of the risk of aspergillosis when managing patients with bronchiectasis, especially those with a history of TB. Such awareness will allow clinicians to make early diagnoses in patients with aspergillosis who benefit from antifungal treatment.

In previous studies, strong associations were observed between Aspergillus infection and NTM-PD. A cross-sectional study reported that among 126 CPA patients, 14.9\% had NTM-PD, making it one of the most common underlying lung diseases [29]. Another case-control study also showed that positivity for Aspergillus serology in patients with bronchiectasis with NTM-PD was 5-fold that in those without NTM-PD [14]. In agreement with these results, we observed that NTM-PD was associated with an increased risk of aspergillosis in patients with bronchiectasis. However, only 1 of 19 patients with NTMPD developed aspergillosis. As the number of patients with NTM-PD was small, our results should be interpreted with caution, and future studies with larger sample sizes are warranted to unveil the link between NTM-PD and increased risk of aspergillosis in patients with bronchiectasis.

ICS use has been regarded as a major risk factor for Aspergillus infection in patients with cystic fibrosis [30-32]. In a recent study, the use of ICS in patients with cystic fibrosis was associated with the isolation of Aspergillus fumigatus in sputum [33]. It has not been clear until now whether the use of ICS increases the risk of aspergillosis in patients with non-cystic fibrosis bronchiectasis. In this study, the use of ICS was associated with an increased risk of incident aspergillosis in univariable analysis; however, the association disappeared after adjustment for covariates in the multivariable analysis. Likewise, the small number of aspergillosis cases might have been related to the statistical insignificance of the use of systemic corticosteroids in patients with bronchiectasis. Thus, future studies are needed to clarify the relationship between corticosteroids use and the development of aspergillosis in patients with bronchiectasis.

In this study, it was necessary to exclude bronchiectasis cases in which aspergillosis was diagnosed within one year after the diagnosis of bronchiectasis (approximately $0.5 \%$ of patients with bronchiectasis) to establish the aspergillosis-naive bronchiectasis cohort. Nevertheless, this finding indicates that some patients with bronchiectasis had coexisting aspergillosis at the time of cohort enrolment. Thus, it is important to develop appropriate clinical strategies to diagnose aspergillosis in patients with newly diagnosed bronchiectasis. However, there is no consensus on this issue. Only the ABPA test for etiologic evaluation has been mentioned in the recently revised British Thoracic Society guidelines for bronchiectasis [34]. Therefore, further studies regarding the evaluation and management of aspergillosis when bronchiectasis is diagnosed are urgently required.

There are several limitations of this study, mostly due to the limitations of the NHIS data related to ICD-10 diagnosis codes. First, we were unable to obtain clinical, radiological, or microbiological information. Second, defining aspergillosis based on ICD-10 codes may underestimate the actual cases of aspergillosis. Third, the incidence of aspergillosis was relatively small, which might have led to a lack of statistical significance in the potential risk factors. 


\section{Conclusions}

The incidence of aspergillosis in patients with bronchiectasis was approximately 5-fold that in patients without bronchiectasis. The risk was higher in the female and younger ( $<60$ years) patients. The risk factors for incident aspergillosis in bronchiectasis were comorbid pulmonary diseases, such as COPD, previous pulmonary TB, and NTM-PD. When managing patients with bronchiectasis, clinicians should consider paying more attention to those with comorbid pulmonary diseases in order to diagnose aspergillosis early and plan effective treatment.

Supplementary Materials: The following are available online at https:/ /www.mdpi.com/article/10 $.3390 / \mathrm{jpm} 11050422 / \mathrm{s} 1$. Table S1: Sensitivity analyses using different washout periods. Subdistribution hazard ratios of aspergillosis in the bronchiectasis cohort relative to the matched cohort. Table S2: Specific diagnostic codes for aspergillosis in the bronchiectasis and matched cohorts. Figure S1: Subgroup analyses of the cumulative incidence (/100,000 person-years) of aspergillosis. (A) Male, (B) Female, (C) Age $<60$ years, (D) Age $\geq 60$ years.

Author Contributions: Conceptualisation, H.C. and H.L.; data curation, J.R., H.Y.P., B.H. and S.-Y.K.; formal analysis, J.R., Y.-S.K. and S.J.L.; funding acquisition, B.Y., T.K., H.C. and H.L.; methodology, J.R., S.W.R., Y.-M.O., J.W.S., H.C. and H.L.; supervision, K.H.C., H.C. and H.L.; visualisation, B.Y., T.K., H.C. and H.L.; writing—original draft, B.Y., T.K., J.R., H.C. and H.L.; writing—review and editing, all authors. All authors have read and agreed to the published version of the manuscript.

Funding: This research was supported by the Korea Medical Device Development Fund grant funded by the Korean government (the Ministry of Science and ICT; the Ministry of Trade, Industry and Energy; the Ministry of Health \& Welfare; the Ministry of Food and Drug Safety) (202014X08-03 to HL), the Basic Science Research Program through the National Research Foundation of Korea funded by the Ministry of Science, Information, and Communications Technologies (2020R1F1A1070468 and 2021M3E5D1A0101517621 to HL and 2019R1G1A1008692 to HC), and grants from the Korean Ministry of Health and Welfare (1520240 to SYK). The funders had no role in the design of the study, collection and analysis of the data, or the preparation of the manuscript.

Institutional Review Board Statement: The study protocol was approved by the Institutional Review Board of Chungbuk National University Hospital (IRB application no. 2020-12-027).

Informed Consent Statement: Not applicable.

Data Availability Statement: The data presented in this study are available upon reasonable request from the corresponding author.

Conflicts of Interest: The authors declare no conflict of interest.

\section{References}

1. Chalmers, J.D.; Chang, A.B.; Chotirmall, S.H.; Dhar, R.; McShane, P.J. Bronchiectasis. Nat. Rev. Dis. Primers 2018, 4, 45. [CrossRef]

2. King, P.T. The pathophysiology of bronchiectasis. Int. J. Chron. Obs. Pulmon. Dis. 2009, 4, 411-419. [CrossRef]

3. Máiz, L.; Vendrell, M.; Olveira, C.; Girón, R.; Nieto, R.; Martínez-García, M. Prevalence and factors associated with isolation of Aspergillus and Candida from sputum in patients with non-cystic fibrosis bronchiectasis. Respir. Int. Rev. Thorac. Dis. 2015, 89, 396-403. [CrossRef]

4. Gashynova, K.; Suska, K.; Dmytrychenko, V. Factors affecting the frequency of exacerbations in adult patients with bronchiectasis. Wiad Lek 2020, 73, 1717-1722. [CrossRef] [PubMed]

5. Mirsaeidi, M.; Hadid, W.; Ericsoussi, B.; Rodgers, D.; Sadikot, R.T. Non-tuberculous mycobacterial disease is common in patients with non-cystic fibrosis bronchiectasis. Int. J. Infect. Dis 2013, 17, e1000-e1004. [CrossRef]

6. Yang, B.; Ryu, J.; Kim, T.; Jo, Y.S.; Kim, Y.; Park, H.Y.; Kang, Y.A.; Lee, S.J.; Lee, O.-J.; Moon, J.-Y.; et al. Impact of bronchiectasis on incident NTM pulmonary disease: A 10-year national cohort study. CHEST 2020. [CrossRef]

7. Denning, D.W.; Pleuvry, A.; Cole, D.C. Global burden of allergic bronchopulmonary aspergillosis with asthma and its complication chronic pulmonary aspergillosis in adults. Med. Mycol. 2013, 51, 361-370. [CrossRef]

8. De Soyza, A.; Aliberti, S. Bronchiectasis and Aspergillus: How are they linked? Med. Mycol. 2017, 55, 69-81. [CrossRef] [PubMed]

9. Kosmidis, C.; Denning, D.W. The clinical spectrum of pulmonary aspergillosis. Thorax 2015, 70, 270-277. [CrossRef]

10. Lee, J.; Lee, J.S.; Park, S.H.; Shin, S.A.; Kim, K. Cohort Profile: The national health insurance service-national sample cohort (NHIS-NSC), South Korea. Int. J. Epidemiol. 2017, 46, e15. [CrossRef] [PubMed] 
11. Choi, H.; Yang, B.; Nam, H.; Kyoung, D.S.; Sim, Y.S.; Park, H.Y.; Lee, J.S.; Lee, S.W.; Oh, Y.M.; Ra, S.W.; et al. Population-based prevalence of bronchiectasis and associated comorbidities in South Korea. Eur. Respir. J. 2019, 54. [CrossRef]

12. Lee, M.R.; Huang, H.L.; Chen, L.C.; Yang, H.C.; Ko, J.C.; Cheng, M.H.; Chong, I.W.; Lee, L.N.; Wang, J.Y.; Dimopoulos, G. Seroprevalence of Aspergillus IgG and disease prevalence of chronic pulmonary aspergillosis in a country with intermediate burden of tuberculosis: A prospective observational study. Clin. Microbiol. Infect. 2020, 26, e1091-e1097. [CrossRef]

13. Andrejak, C.; Nielsen, R.; Thomsen, V.O.; Duhaut, P.; Sorensen, H.T.; Thomsen, R.W. Chronic respiratory disease, inhaled corticosteroids and risk of non-tuberculous mycobacteriosis. Thorax 2013, 68, 256-262. [CrossRef] [PubMed]

14. Kunst, H.; Wickremasinghe, M.; Wells, A.; Wilson, R. Nontuberculous mycobacterial disease and Aspergillus-related lung disease in bronchiectasis. Eur. Respir. J. 2006, 28, 352-357. [CrossRef]

15. Dhar, R.; Singh, S.; Talwar, D.; Mohan, M.; Tripathi, S.K.; Swarnakar, R.; Trivedi, S.; Rajagopala, S.; D'Souza, G.; Padmanabhan, A.; et al. Bronchiectasis in India: Results from the European multicentre bronchiectasis audit and research collaboration (EMBARC) and respiratory research network of India registry. Lancet Glob. Health 2019, 7, e1269-e1279. [CrossRef]

16. Visser, S.K.; Bye, P.T.P.; Fox, G.J.; Burr, L.D.; Chang, A.B.; Holmes-Liew, C.L.; King, P.; Middleton, P.G.; Maguire, G.P.; Smith, D.; et al. Australian adults with bronchiectasis: The first report from the Australian bronchiectasis registry. Respir. Med. 2019, 155, 97-103. [CrossRef] [PubMed]

17. Araújo, D.; Shteinberg, M.; Aliberti, S.; Goeminne, P.C.; Hill, A.T.; Fardon, T.C.; Obradovic, D.; Stone, G.; Trautmann, M.; Davis, A.; et al. The independent contribution of Pseudomonas aeruginosa infection to long-term clinical outcomes in bronchiectasis. Eur. Respir. J. 2018, 51. [CrossRef] [PubMed]

18. Salzer, H.J.; Heyckendorf, J.; Kalsdorf, B.; Rolling, T.; Lange, C. Characterization of patients with chronic pulmonary aspergillosis according to the new ESCMID/ERS/ECMM and IDSA guidelines. Mycoses 2017, 60, 136-142. [CrossRef] [PubMed]

19. Iqbal, N.; Irfan, M.; Zubairi, A.B.; Jabeen, K.; Awan, S.; Khan, J.A. Clinical manifestations and outcomes of pulmonary aspergillosis: Experience from Pakistan. BMJ Open Respir. Res. 2016, 3, e000155. [CrossRef]

20. Global Strategy for the Diagnosis, Management, and Prevention of Chronic Obstructive Pulmonary Disease (2021 Report). Available online: https:/ / goldcopd.org/2021-gold-reports (accessed on 5 March 2021).

21. Shahi, M.; Ayatollahi Mousavi, S.A.; Nabili, M.; Aliyali, M.; Khodavaisy, S.; Badali, H. Aspergillus colonization in patients with chronic obstructive pulmonary disease. Curr. Med. Mycol. 2015, 1, 45-51. [CrossRef] [PubMed]

22. Waqas, S.; Dunne, K.; Talento, A.F.; Wilson, G.; Martin-Loeches, I.; Keane, J.; Rogers, T.R. Prospective observational study of respiratory Aspergillus colonization or disease in patients with various stages of chronic obstructive pulmonary disease utilizing culture versus nonculture techniques. Med. Mycol. 2020. [CrossRef]

23. Saraceno, J.L.; Phelps, D.T.; Ferro, T.J.; Futerfas, R.; Schwartz, D.B. Chronic necrotizing pulmonary aspergillosis: Approach to management. Chest 1997, 112, 541-548. [CrossRef] [PubMed]

24. Camuset, J.; Nunes, H.; Dombret, M.C.; Bergeron, A.; Henno, P.; Philippe, B.; Dauriat, G.; Mangiapan, G.; Rabbat, A.; Cadranel, J. Treatment of chronic pulmonary aspergillosis by voriconazole in nonimmunocompromised patients. Chest 2007, 131, 1435-1441 [CrossRef]

25. Tuberculosis Association. Aspergilloma and residual tuberculous cavities-The results of a resurvey. Tubercle 1970, 51, 227-245. [CrossRef]

26. Denning, D.W.; Pleuvry, A.; Cole, D.C. Global burden of chronic pulmonary aspergillosis as a sequel to pulmonary tuberculosis. Bull. World Health Organ. 2011, 89, 864-872. [CrossRef] [PubMed]

27. Sehgal, I.S.; Dhooria, S.; Prasad, K.T.; Muthu, V.; Aggarwal, A.N.; Rawat, A.; Pal, A.; Bal, A.; Garg, M.; Chakrabarti, A.; et al. Sensitization to A.fumigatus in subjects with non-cystic fibrosis bronchiectasis. Mycoses 2020. [CrossRef]

28. Page, I.D.; Byanyima, R.; Hosmane, S.; Onyachi, N.; Opira, C.; Richardson, M.; Sawyer, R.; Sharman, A.; Denning, D.W. Chronic pulmonary aspergillosis commonly complicates treated pulmonary tuberculosis with residual cavitation. Eur. Respir. J. 2019, 53, 1801184. [CrossRef] [PubMed]

29. Smith, N.L.; Denning, D.W. Underlying conditions in chronic pulmonary aspergillosis including simple aspergilloma. Eur. Respir. J. 2011, 37, 865-872. [CrossRef]

30. Ritz, N.; Ammann, R.A.; Aebischer, C.C.; Schoeni-Affolter, F.; Schoeni, M.H. Risk factors for allergic bronchopulmonary aspergillosis and sensitisation to Aspergillus fumigatus in patients with cystic fibrosis. Eur. J. Pediatrics 2005, 164, 577-582. [CrossRef]

31. Noni, M.; Katelari, A.; Dimopoulos, G.; Kourlaba, G.; Spoulou, V.; Alexandrou-Athanassoulis, H.; Doudounakis, S.-E.; TzoumakaBakoula, C. Inhaled corticosteroids and Aspergillus fumigatus isolation in cystic fibrosis. Med. Mycol. 2014, 52, 715-722. [CrossRef] [PubMed]

32. Hong, G.; Psoter, K.J.; Jennings, M.T.; Merlo, C.A.; Boyle, M.P.; Hadjiliadis, D.; Kawut, S.M.; Lechtzin, N. Risk factors for persistent Aspergillus respiratory isolation in cystic fibrosis. J. Cyst. Fibros. 2018, 17, 624-630. [CrossRef] [PubMed]

33. Hong, G.; Alby, K.; Ng, S.C.W.; Fleck, V.; Kubrak, C.; Rubenstein, R.C.; Dorgan, D.J.; Kawut, S.M.; Hadjiliadis, D. The presence of Aspergillus fumigatus is associated with worse respiratory quality of life in cystic fibrosis. J. Cyst. Fibros. 2020, 19, 125-130. [CrossRef] [PubMed]

34. Hill, A.T.; Welham, S.A.; Sullivan, A.L.; Loebinger, M.R. Updated BTS adult bronchiectasis guideline 2018: A multidisciplinary approach to comprehensive care. Thorax 2019, 74, 1-3. [CrossRef] [PubMed] 